\title{
Erratum: Succinct Non-interactive Arguments via Linear Interactive Proofs
}

\author{
Nir Bitansky ${ }^{1}$, Alessandro Chiesa ${ }^{2}$, Yuval Ishai ${ }^{3}$, \\ Omer Paneth $^{4}$, and Rafail Ostrovsky ${ }^{5}$ \\ ${ }^{1}$ Tel Aviv University \\ ${ }^{2}$ MIT \\ ${ }^{3}$ Technion \\ ${ }^{4}$ Boston University \\ ${ }^{5}$ UCLA
}

\section{DOI 10.1007/978-3-642-36594-2_41}

Unfortunately the order of appearance of the authors on the title page is not correct. Second last and last author were switched. In fact, the authors should be listed in alphabetical order so that Rafail Ostrovsky is second last and Omer Paneth is last author.

The correct order is:

Nir Bitansky, Alessandro Chiesa, Yuval Ishai, Rafail Ostrovsky, and Omer Paneth 